\title{
Years of life gained by multifactorial intervention in patients with type 2 diabetes mellitus and microalbuminuria: 21 years follow-up on the Steno-2 randomised trial
}

\author{
Peter Gæde $^{1,2}$ - Jens Oellgaard ${ }^{1,2,3}$ - Bendix Carstensen ${ }^{3}$ - Peter Rossing ${ }^{3,4,5}$. \\ Henrik Lund-Andersen ${ }^{3,5,6} \cdot$ Hans-Henrik Parving ${ }^{5,7} \cdot$ Oluf Pedersen $^{8}$
}

Received: 7 April 2016 / Accepted: 1 July 2016/Published online: 16 August 2016

(C) The Author(s) 2016. This article is published with open access at Springerlink.com

\begin{abstract}
Aims/hypothesis The aim of this work was to study the potential long-term impact of a 7.8 years intensified, multifactorial intervention in patients with type 2 diabetes mellitus and microalbuminuria in terms of gained years of life and years free from incident cardiovascular disease.

Methods The original intervention (mean treatment duration 7.8 years) involved 160 patients with type 2 diabetes and microalbuminuria who were randomly assigned (using sealed envelopes) to receive either conventional therapy or intensified, multifactorial treatment including both behavioural and
\end{abstract}

Peter Gæde and Jens Oellgaard contributed equally to this paper.

Electronic supplementary material The online version of this article (doi:10.1007/s00125-016-4065-6) contains peer-reviewed but unedited supplementary material, which is available to authorised users.

Oluf Pedersen

oluf@sund.ku.dk

1 Department of Cardiology and Endocrinology, Slagelse Hospital, Slagelse, Denmark

2 Institute for Regional Health Research, University of Southern Denmark, Odense, Denmark

3 Steno Diabetes Center, Gentofte, Denmark

4 Faculty of Health, Aarhus University, Aarhus, Denmark

5 Faculty of Health Sciences, University of Copenhagen, Copenhagen, Denmark

6 Capital Region Eye Clinic, Copenhagen, Denmark

7 Department of Medical Endocrinology, Rigshospitalet, Copenhagen, Denmark

8 Novo Nordisk Foundation Center for Basic Metabolic Research, Section of Metabolic Genetics, Faculty of Health and Medical Sciences, University of Copenhagen, Universitetsparken 1, DK-2100 Copenhagen, Denmark pharmacological approaches. After 7.8 years the study continued as an observational follow-up with all patients receiving treatment as for the original intensive-therapy group. The primary endpoint of this follow-up 21.2 years after intervention start was difference in median survival time between the original treatment groups with and without incident cardiovascular disease. Non-fatal endpoints and causes of death were adjudicated by an external endpoint committee blinded for treatment allocation.

Results Thirty-eight intensive-therapy patients vs 55 conventional-therapy patients died during follow-up (HR 0.55 [95\% CI 0.36, 0.83], $p=0.005$ ). The patients in the intensivetherapy group survived for a median of 7.9 years longer than the conventional-therapy group patients. Median time before first cardiovascular event after randomisation was 8.1 years longer in the intensive-therapy group $(p=0.001)$. The hazard for all microvascular complications was decreased in the intensivetherapy group in the range 0.52 to 0.67 , except for peripheral neuropathy (HR 1.12).

Conclusions/interpretation At 21.2 years of follow-up of 7.8 years of intensified, multifactorial, target-driven treatment of type 2 diabetes with microalbuminuria, we demonstrate a median of 7.9 years of gain of life. The increase in lifespan is matched by time free from incident cardiovascular disease.

Trial registration: ClinicalTrials.gov registration no. NCT00320008.

Funding: The study was funded by an unrestricted grant from Novo Nordisk A/S.

Keywords Albuminuria $\cdot$ Cardiovascular disease $\cdot$ Diabetes complications · Diabetes mellitus, type 2 - Diabetic nephropathy $\cdot$ Diabetic neuropathy $\cdot$ Diabetic retinopathy Follow-up studies $\cdot$ Humans 


\section{Abbreviation}

CVD Cardiovascular disease

\section{Introduction}

Type 2 diabetes mellitus is a complex disorder often featuring adiposity, hypertension, dyslipidaemia and increased blood platelet aggregation in addition to hyperglycaemia, giving rise to an increased risk of macro- and microvascular damage and reduced life expectancy. Over the past two decades, a number of single-risk-factor intervention and post-trial studies have provided evidence for recommending multifactorial treatment of this common disorder (as reviewed in [1]). Still, patients with type 2 diabetes mellitus have increased risk for early mortality [2] and may suffer from multiple organ damage with recurrent event rates of up to $15 \%$ per year during follow-up of various trials [3-8] even though recent surveys suggest favourable changes in diabetes-related complications including death $[9,10]$.

Therefore, this follow-up, a randomised trial of intensified vs standard multifactorial intervention for 7.8 years in patients with type 2 diabetes mellitus and microalbuminuria, was designed to address the median differences in lifespan with and without incident cardiovascular events between the originally intensified vs conventionally treated patients. We use the term 'years of life gained' to emphasise the decreased life expectancy in patients with type 2 diabetes mellitus and microalbuminuria.

In the present follow-up study the patients were observed for a median of 13.2 years after the formal interventional part of the study ended.

\section{Methods}

Study design The Steno-2 study was initiated in 1993, enrolling 160 Danish patients of European descent with type 2 diabetes mellitus and microalbuminuria [11-13]. The design and methods for patient inclusion, randomisation, treatment and initial follow-up have been reported in detail previously [11-13].

Briefly, patients were randomised 1:1, stratified in blocks by sex, age, known diabetes duration and urinary albumin excretion rate $(<100 \mathrm{mg} /$ day vs $>100 \mathrm{mg} /$ day $)$ using sealed envelopes, to receive either conventional multifactorial treatment with treatment goals at all times according to existing national guidelines or to receive intensified, multifactorial treatment targeting co-existing risk factors for late diabetic complications. Eighty patients were assigned to each group. Conventional-therapy patients were followed by their general practitioner, but at all times had the opportunity of being referred to specialist treatment. The treatment in the intensivetherapy group at a specialised diabetes clinic was target-driven with stepwise implementation of both behavioural and pharmacological treatment following a structured approach. Treatment goals for the groups are shown in Section 1 of the electronic supplementary material (ESM) in ESM Table 1.

Patients completed up to six study visits at Steno Diabetes Center, namely at baseline and after an average of 1.9, 3.8, 7.8 and 13.3 years, respectively, and at the termination visit after 21.2 years.

Study population Of the 160 patients originally enrolled in the Steno-2 study, 130 completed the interventional part of the study. Patient flow is depicted in Fig. 1. All patients completing the interventional part of the study provided written informed consent to continue participation in the observational post-trial follow-up. In the entire follow-up period, one patient in the conventional group and none in the intensive group were lost to follow-up. The loss was due to emigration and data collection on that patient terminated in 2007. All other patients were followed to death or study termination.

Due to ethical concerns, following the marked risk reductions found with intensive therapy after 7.8 years, all patients were subsequently offered intensified multifactorial treatment according to the original protocol using targets resembling current EASD/ESC and ADA guidelines [14, 15].

The protocol for the follow-up trial was in accordance with the declaration of Helsinki and approved by the local ethics committee (Ethics committee, Capital Region of Denmark; protocol ID number: HKA-99035-GS, add. 41104) and by the Danish Data Protection Agency (J.Nr. 2015-41-4042).

Procedures, measurements and endpoints The follow-up investigations were initiated to investigate the difference in median time to $50 \%$ mortality in each of the two original treatment groups. This follow-up study was done as an amendment to the original study protocol.

The primary endpoint for the present study was the difference between the two treatment groups in the survival time after randomisation without and with cardiovascular disease (CVD). The secondary endpoint was defined as a composite of cardiovascular events (time to incident CVD and number of cardiovascular events) as well as mortality and CVD rates. The tertiary endpoints were rate of incident diabetic nephropathy, rate of end-stage renal disease and the development or progression of diabetic retinopathy and neuropathy.

For the follow-up examinations, all living patients were invited to an outpatient clinic visit assessing anthropometric, biochemical and physiological variables as well as detailed status on micro- and macrovascular complications. The examinations were conducted by a single laboratory technician who was unaware of the original randomisation assignments. 
Fig. 1 Consort diagram of patient flow throughout the entire observation period. Procedures for enrolment and randomisation are described in [11]. Numbers lost to follow-up are cumulative

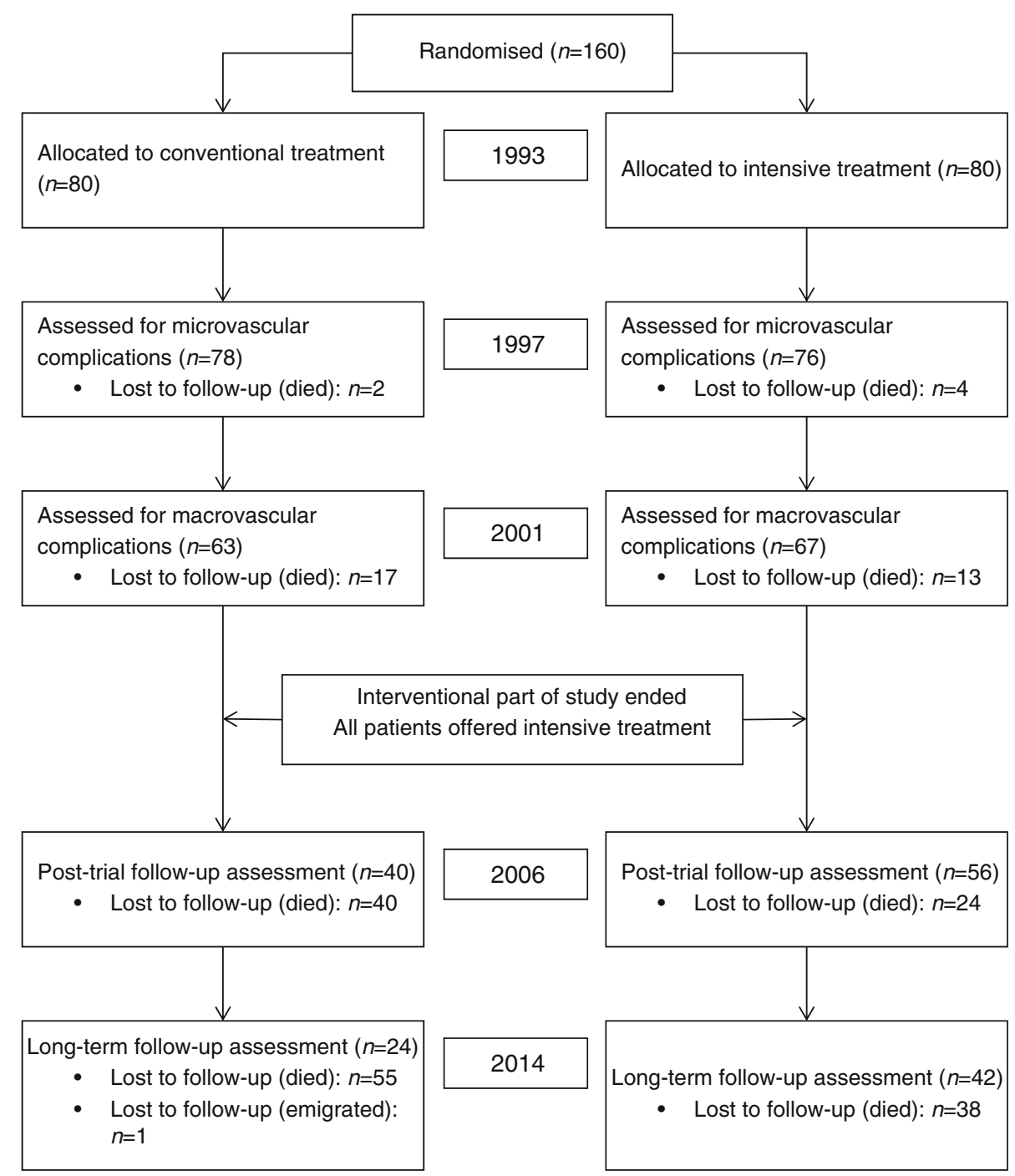

Supplementary clinical evaluations were collected from nationwide registries when not obtainable at the study visit. All measurement methods were in accordance with previous follow-up protocols [13].

For endpoint assessment, all patients were tracked by their unique, static Central Personal Registration number using the Danish Civil Registration System. All hospital contacts diagnosed to possibly fulfil the pre-specified criteria (see ESM Section 2) or relating to the criteria (including ICD-10 [www.who.int/classifications/icd/en/] observation-diagnoses [DZ0.3x] and symptom-diagnoses [e.g. DR0.74, chest pain]) were extracted from the Danish National Patient Registry and used as cases for the endpoint committee. Complete health records for cases were obtained from nationwide electronic health records and adjudicated for endpoints by an external committee masked for patients' original treatment allocation. The registries have proven highly valid and complete [16, 17$]$. Hospitals report mandatorily to the registries and the reported data on diagnoses and procedures translates directly to the funding of the hospitals. The data provide information from 1978 onwards. Hospitals mandatorily keep individual patient records related to hospitalisations and outpatient treatment for at least 10 years after the last record.

Cardiovascular events were defined as a composite of death from cardiovascular causes, myocardial infarction, stroke, amputation due to ischaemia and cardiac or peripheral revascularisation. If an event occurred as a direct consequence of, or as an adverse event related to another, immediately prior event (e.g. percutaneous transluminary coronary angioplasty immediately after acute myocardial infarction; see ESM section 2.1), this latter event was not considered in the analyses of separate endpoints. Self-reported cardiovascular events prior to randomisation were not considered. Microvascular complications were defined, measured and evaluated as described in ESM Section 3.

Statistical analyses All statistical analyses were conducted using the intention-to-treat principle. Adherence to the 
treatment was assessed at study visits in both groups based on interviews with patients.

Overall cumulative survival, as well as CVD-free survival, by time since randomisation was calculated for the two treatment groups by the Kaplan-Meier estimator. Median survival time from randomisation was compared between the treatment groups. CIs for the medians were calculated using bootstrapping. We specifically chose difference in median survival time between groups to make a clear statement in easily understandable terms in addition to the more extensively used, but more difficult to interpret, HR and RR reduction.

Proportional hazards Poisson models were fitted for mortality (both from cardiovascular causes and from other causes) and cardiovascular events, taking treatment group, age, sex and current CVD status into account, using smooth underlying hazards (time since baseline). Current CVD status was included in models for all-cause mortality as $0,1,2$ or 3 or more cardiovascular events after randomisation. Thus, we modelled both death and (extra) CVD events as outcome, and the models were used to compute the average years of life lived and the years of life free of CVD during the study period and thereby the average number of years gained by intensive treatment. Models were checked for proportional hazards between randomisation groups by likelihood-ratio tests. Diabetes duration at baseline, as well as the interaction with treatment allocation, was included in a model to assess the effect of diabetes duration on mortality.

Except for end-stage renal disease (where exact date of transplantation or first dialysis treatment was known), the status of microvascular complications was only assessed at study visits, and the exact event date is therefore unknown. For analysis purposes a random date between the last day without and first day with the specified complication was imputed as the event date. When the progression between states jumped more than one category (e.g. from EURODIAB-score 2 to 5 between two observation points), random dates between the two observations for the steps were generated and used in analyses of transition rates. Sensitivity analyses were performed by repeating the random allocation of dates.

Transition rates between states and group comparison were analysed as for cardiovascular events. For each of the four types of outcome (retinopathy, autonomic neuropathy, peripheral neuropathy and albuminuria) we estimated the rates of transition between states of increasing severity and used these to construct plots showing the fraction of patients in each state at different times after randomisation.

A fuller account of statistical approaches and detailing of analyses can be found in ESM Sections 4-7. For the anthropometric, biochemical and physiological variables, the $t$ test, Mann-Whitney $U$ test and $\chi^{2}$ statistics were applied for the comparison of means, medians and proportions, respectively. In the results section all estimates are followed by $95 \%$ CIs in parentheses. Analyses were conducted using R, version 3.3.0, using the Epi package version 2.5 or Stata/IC version 14.1 for Windows (StataCorp LP, College Station, TX, USA).

\section{Results}

Forty-two patients in the original intensive-therapy group and 24 in the original conventional-therapy group completed the entire follow-up period. Median observation time for patients completing follow-up was 21.2 years (range 20.2-21.9 years). Demographic, anthropometric, clinical variables and prescribed drug intake are presented in Table 1.

Mortality At the end of follow-up, 38 (48\%) of the original intensive-therapy patients had died compared with $55(69 \%)$ of conventional-therapy patients. Median survival time in the conventional-therapy group was 13.3 years, and the difference in median survival time after randomisation was therefore at least 7.9 years (95\% CI 2.2, 9.6 years) (Fig. 2a). However, formally we were not able to calculate median survival time after randomisation in the intensive-therapy group (since $48 \%$ [and not 50\% as required] of intensive-therapy group patients died before the end of follow-up) and the calculated differences in median survival might thus underestimate the real difference.

The observed median time to first CVD event or death was 8.0 years in the conventional-therapy group and 16.1 years in the intensive-therapy group; a difference of 8.1 years $(95 \% \mathrm{CI}$ 4.0, 12.6 years) (Fig. 2b).

HR $(95 \% \mathrm{CI})$ estimates for secondary and tertiary endpoints are shown in Fig. 3. The overall, adjusted mortality rate in the original intensive-therapy group was reduced by $45 \%$ during the entire follow-up period. The absolute risk reduction in mortality in the intensive-therapy group was $21 \%$.

Death from cardiovascular causes was reduced by $62 \%$ in the intensive-therapy group and no difference in noncardiovascular mortality between groups was observed. No evidence that the mortality rate in the original conventionaltherapy group increased after treatment intensification at 7.8 years was found (ESM Fig. 1); however, the CVD mortality rates were not proportional (the HR for intensive vs conventional were higher during the first 5 years after randomisation and lower later on). Following neutralisation of the intervention after 7.8 years, the rate-ratio for all-cause mortality was significantly in favour of the original intensive-therapy group.

We found a trend towards an effect of diabetes duration on mortality (HR 1.03 per year [95\% CI 1.00, 1.07], $p=0.074$; ESM Fig. 2). This association was equal for the two groups $(p=0.29)$.

Cardiovascular disease In the intensive-therapy group, 35 patients experienced a cardiovascular event compared with 51 patients in the conventional-therapy group, corresponding to a $51 \%$ RR reduction and $20 \%$ absolute risk reduction for incident 
Table 1 Clinical, anthropometric and biochemical data and self-reported use of prescribed drugs

\begin{tabular}{|c|c|c|c|c|c|c|}
\hline \multirow[t]{2}{*}{ Variable } & \multicolumn{2}{|c|}{ Baseline (1993) } & \multicolumn{2}{|c|}{ End of intervention (2001) } & \multicolumn{2}{|c|}{ End of follow-up (2014) } \\
\hline & $\begin{array}{l}\text { Intensive } \\
(N=80)\end{array}$ & $\begin{array}{l}\text { Standard } \\
(N=80)\end{array}$ & $\begin{array}{l}\text { Intensive } \\
(N=67)\end{array}$ & $\begin{array}{l}\text { Standard } \\
(N=63)\end{array}$ & $\begin{array}{l}\text { Intensive } \\
(N=42)\end{array}$ & $\begin{array}{l}\text { Standard } \\
(N=24)\end{array}$ \\
\hline Age, years & $54.9 \pm 7.2$ & $55.2 \pm 7.2$ & $66.0 \pm 7.0$ & $66.1 \pm 6.8$ & $72.1 \pm 6.4$ & $71.9 \pm 5.8$ \\
\hline Age range, years & $37-67$ & $42-67$ & $50-80$ & $55-80$ & $58-83$ & $63-86$ \\
\hline Proportion male sex, $\%$ & 79 & 70 & 78 & 72 & 70 & 67 \\
\hline Diabetes duration, years & $4(0-30)$ & $6(0-29)$ & $12(7-28)$ & $14(7-37)$ & $25(20-41)$ & $27(21-51)$ \\
\hline \multicolumn{7}{|l|}{ BMI, $\mathrm{kg} / \mathrm{m}^{2}$} \\
\hline Men & $29.3 \pm 3.6$ & $30.3 \pm 5.3$ & $30.0 \pm 4.3$ & $30.8 \pm 5.6$ & $29.8 \pm 4.4$ & $31 \pm 5.4$ \\
\hline Women & $31.1 \pm 4.5$ & $28.9 \pm 3.8$ & $33.8 \pm 6.8$ & $30.0 \pm 4.4$ & $31.5 \pm 6.4$ & $31.8 \pm 3.8$ \\
\hline \multicolumn{7}{|l|}{ Waist circumference, $\mathrm{cm}$} \\
\hline Men & $105 \pm 10$ & $107 \pm 14$ & $108 \pm 10$ & $112 \pm 14$ & $109 \pm 12$ & $110 \pm 15$ \\
\hline Women & $100 \pm 14$ & $101 \pm 13$ & $108 \pm 14$ & $107 \pm 11$ & $108 \pm 11$ & $112 \pm 6$ \\
\hline \multicolumn{7}{|l|}{ Blood pressure, $\mathrm{mmHg}$} \\
\hline Systolic & $146 \pm 11$ & $149 \pm 19$ & $131 \pm 13$ & $146 \pm 18^{*}$ & $145 \pm 19$ & $143 \pm 18$ \\
\hline Diastolic & $85 \pm 10$ & $86 \pm 11$ & $73 \pm 11$ & $78 \pm 10 *$ & $70 \pm 10$ & $68 \pm 11$ \\
\hline Fasting glucose, mmol/l & $10.1 \pm 3.1$ & $10.5 \pm 3.0$ & $7.2 \pm 2.5$ & $9.9 \pm 3.9 *$ & $7.7 \pm 2.5$ & $8.4 \pm 2.7$ \\
\hline \multicolumn{7}{|l|}{$\mathrm{HbA}_{1 \mathrm{c}}$} \\
\hline IFCC, $\mathrm{mmol} / \mathrm{mol}$ & $68 \pm 6$ & $73 \pm 5$ & $63 \pm 10$ & $75 \pm 4 *$ & $58 \pm 15$ & $59 \pm 12$ \\
\hline DCCT, $\%$ & $8.4 \pm 2.7$ & $8.8 \pm 2.6$ & $7.9 \pm 3.1$ & $9.0 \pm 2.5$ & $7.4 \pm 1.4$ & $7.5 \pm 1.2$ \\
\hline Stimulated C-peptide, $\mathrm{pmol} / \mathrm{l}^{\mathrm{a}}$ & 1438 & 1514 & 1140 & 1090 & 912 & 1059 \\
\hline Total cholesterol, mmol/l & $5.4 \pm 1.1$ & $6.0 \pm 1.3$ & $4.1 \pm 0.9$ & $5.6 \pm 1.3^{*}$ & $3.9 \pm 0.9$ & $4.1 \pm 1.1$ \\
\hline HDL-cholesterol, mmol/1 & $1.0 \pm 0.2$ & $1.0 \pm 0.3$ & $1.2 \pm 0.4$ & $1.2 \pm 0.3$ & $1.2 \pm 0.5$ & $1.1 \pm 0.3$ \\
\hline LDL-cholesterol, mmol/1 & $3.4 \pm 0.9$ & $3.5 \pm 1.0$ & $2.1 \pm 0.8$ & $3.3 \pm 0.9 *$ & $2.1 \pm 0.8$ & $2.1 \pm 0.8$ \\
\hline Triacylglycerol, mmol/1 & $1.8(1.3-2.7)$ & $2.5(1.4-3.2)$ & $1.3(0.9-2.3)$ & $1.8(1.3-3.2)^{*}$ & $1.2(0.8-1.7)$ & $1.4(0.9-2.4)$ \\
\hline p-Creatinine, $\mu \mathrm{mol} / 1$ & $78 \pm 17$ & $76 \pm 16$ & $102 \pm 32$ & $111 \pm 85$ & $108 \pm 78$ & $137 \pm 119$ \\
\hline $\mathrm{u}-\mathrm{AER}, \mathrm{mg} / 24 \mathrm{~h}$ & $78(61-120)$ & $69(47-113)$ & $46(17-201)$ & $126(38-547)^{*}$ & $98(38-309)$ & $74(18-377)$ \\
\hline \multicolumn{7}{|l|}{ Self-reported drug intake (\%) } \\
\hline No glucose-lowering drugs & 35 & 26 & 1 & 6 & 2 & 0 \\
\hline Metformin & 13 & 19 & 50 & 34 & 59 & 50 \\
\hline Insulin & 6 & 14 & 57 & 54 & 71 & 79 \\
\hline Any non-insulin hypoglycaemic & 59 & 61 & 74 & 61 & 85 & $58^{*}$ \\
\hline $\begin{array}{l}\text { Insulin + other hypoglycaemic } \\
\text { drug }\end{array}$ & 0 & 1 & 32 & 21 & 51 & 50 \\
\hline $\mathrm{ACE}$ inhibitor or $\mathrm{ARB}$ & 20 & 19 & 97 & $70^{*}$ & 76 & 92 \\
\hline Diuretic & 21 & 28 & 58 & 60 & 68 & 83 \\
\hline Calcium antagonist & 14 & 6 & 36 & 29 & 51 & 46 \\
\hline Beta blocker & 10 & 1 & 19 & 16 & 34 & 42 \\
\hline Other antihypertensive drug & 1 & 1 & 4 & 6 & 24 & 25 \\
\hline Any antihypertensive drug & 41 & 41 & 99 & $83^{*}$ & 93 & 100 \\
\hline Statin & 0 & 3 & 85 & $22^{*}$ & 80 & 92 \\
\hline Fibrate & 1 & 1 & 1 & 5 & 5 & 0 \\
\hline Antiplatelet therapy & 16 & 15 & 87 & $56^{*}$ & 66 & 83 \\
\hline
\end{tabular}

Data are means $\pm \mathrm{SD}$ or medians (interquartile range) unless stated otherwise

${ }^{a}$ Median

${ }^{*} p<0.05$ for difference between groups

$\mathrm{u}-\mathrm{AER}$, urinary albumin excretion rate; $\mathrm{ARB}$, angiotensin receptor blocker 
a

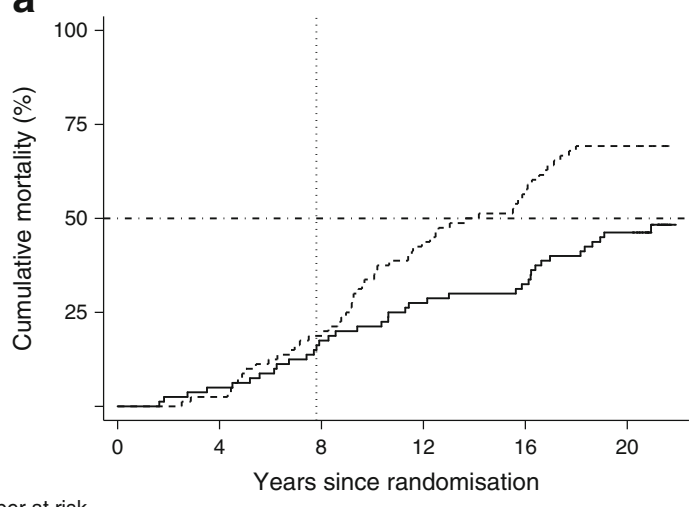

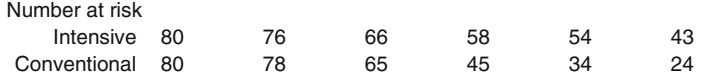

b

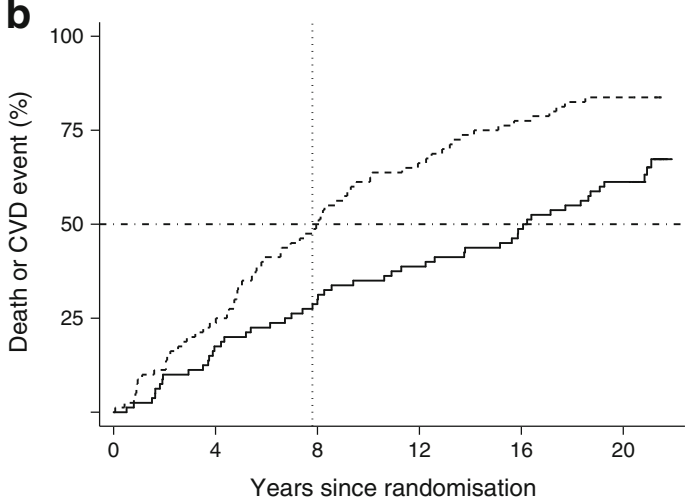

Number at risk

Intensive 80

Conventional 80

66

56
40

49
27

41
18

13
Fig. 2 Cumulative mortality (a) and cumulative incidence of the composite cardiovascular or death endpoint (b). Solid lines, patients in the intensive-therapy group; dashed lines, patients in the conventional-therapy group; vertical dotted lines, end of trial and start of intensification of conventional-therapy group patients' treatment; horizontal dashed lines intersect with survival curves at median survival time (a) and median CVD-free survival time (b). The median survival time in the original intensive-therapy group was at least 7.9 years longer than in the conventional-therapy group ( $48 \%$ of patients in the intensive-therapy group died during follow-up, so formally this might be an underestimate, since $50 \%$ mortality is required to calculate the median). The median difference in survival before first CVD event was 8.1 years in favour of the original intensive-therapy group

CVD. Transitions between, and time in, different states of CVD $(0,1,2$ or 3 or more events since randomisation, respectively) are shown in ESM Fig. 5. Twenty-eight patients (35\%) in the intensive group vs $13(16 \%)$ in the conventional group completed the entire follow-up without any incident macrovascular events; HR for CVD event in the intensive-therapy group 0.55 (95\% CI 0.39, 0.77; $p<0.001$ ).

Patients in both groups with one post-baseline cardiovascular event had a higher mortality rate than patients without; HR 3.08 $(95 \%$ CI $1.82,5.19)$ and an almost linear increase in mortality of $2.08(95 \%$ CI $1.73,2.51)$ per extra event. A similar pattern was seen for further CVD events. When the hazard for mortality was adjusted for CVD status, there was no difference in mortality between groups (HR 0.83 [95\% CI 0.54, 1.30], $p=0.43$ ). Thus, the reduced mortality was primarily due to reduced risk of CVD.

The patients in the intensive group experienced a total of 90 cardiovascular events vs 195 events in the conventional group. Nineteen intensive-group patients $(24 \%)$ vs 34 conventional-group patients $(43 \%)$ experienced more than one cardiovascular event. No significant between-group difference in the distribution of specific cardiovascular firstevent types was observed (Table 2 and Fig. 4).

Microvascular complications Hazard rates of progression rates in microvascular complications compared with baseline status are shown Fig. 3. Sensitivity analyses showed a negligible effect of the random dates imputation.

Progression of retinopathy was decreased by $33 \%$ in the intensive-therapy group (Fig. 5). Blindness in at least one eye was reduced in the intensive-therapy group with an HR of 0.47 (95\% CI $0.23,0.98, p=0.044)$. Autonomic neuropathy was decreased by $41 \%$ in the intensive-therapy group (Fig. 5). We observed no difference between groups in the progression of peripheral neuropathy (Fig. 5). Progression to diabetic nephropathy (macroalbuminuria) was reduced by $48 \%$ in the intensive-therapy group (Fig. 5). Ten patients in the conventional-therapy groups vs five patients in the intensivetherapy group progressed to end-stage renal disease $(p=0.061)$.

\section{Discussion}

In the current report from the Steno-2 study we demonstrate that intensified treatment for 7.8 years was associated with a 7.9 years longer median lifespan over a period of 21.2 years follow-up. Furthermore, the increased lifespan was matched by the years gained free from incident CVD. The reduced mortality was caused by a decreased risk of incident CVD and cardiovascular mortality.

Absolute risk and RR reductions for all endpoints were well in line with earlier reported findings, confirming the durability of the intensified, multifactorial approach [13].

The frequency of recurrent events was high in both groups, but patients in the original conventional-therapy group experienced more than twice as many cardiovascular events per person than patients from the original intensive-therapy group. Only a few studies have reported results on recurrent events; none of these have been exclusively in patients with type 2 diabetes $[2,3]$ and the follow-up periods were much shorter, hence direct comparison is difficult.

In the Steno-2 study, we observed high rates of progression for microvascular complications with the vast majority of patients progressing in one or more complication types. Yet, we found significant and clinically relevant risk reductions for autonomic neuropathy, nephropathy, and retinopathy, as well as blindness, and a trend towards reduced risk for end-stage renal 


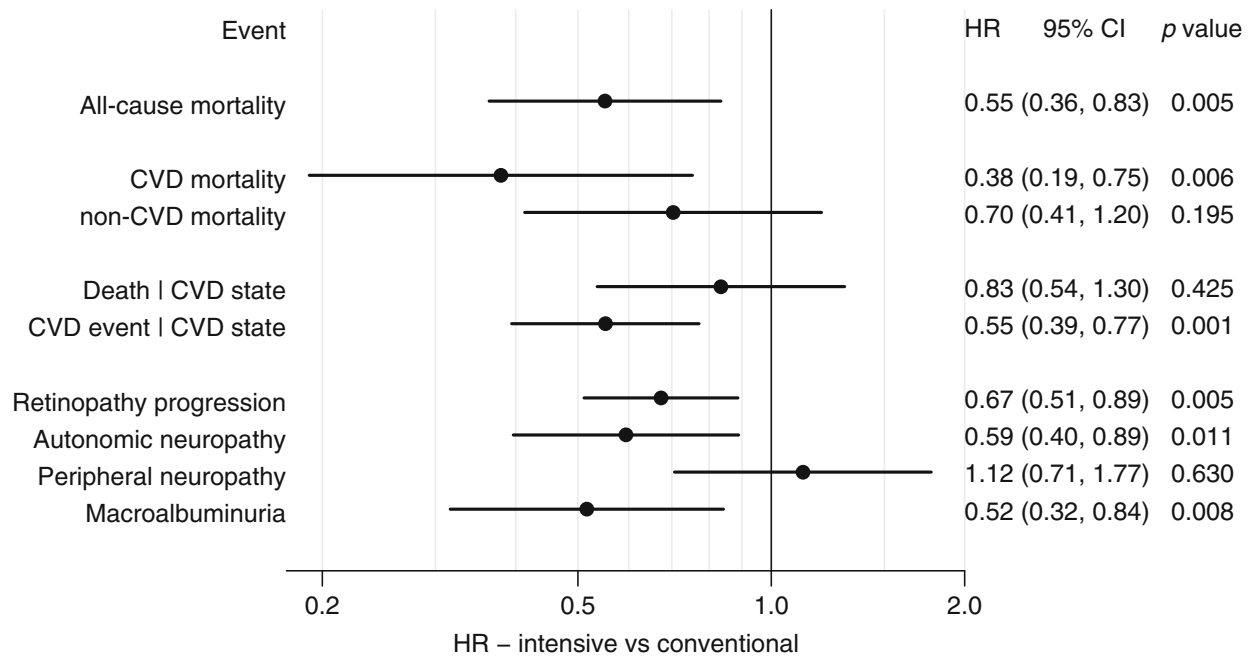

Fig. 3 Forest plot of the HR (95\% CI) for secondary and tertiary endpoints. Intensive vs conventional treatment. We found significant risk reductions for all-cause mortality, CVD mortality, CVD events and progression of retinopathy, autonomic neuropathy and macroalbuminuria.

disease. Retinopathy grading was not corrected for cataract development and surgery, which made the longitudinal comparison less exact. For the rarer and later-onset complications (i.e. end-stage renal disease and blindness) competing risk from death might be a serious issue, underestimating the true effect of the intervention.

The risk reductions reported are high compared with those reported in single-risk-factor intervention trials [18-21]. Concomitant treatment of multiple risk factors seems to be of profound importance. A recent report from VADT (Veterans Affairs Diabetes Trial) demonstrated larger risk reductions in patients with diabetes mellitus achieving both $\mathrm{HbA}_{1 \mathrm{c}}$ and LDLcholesterol goals compared with patients achieving only one of these goals [22]. Similar findings have been reported from the ADVANCE (Action in Diabetes and Vascular Disease: Preterax
No difference was observed for non-CVD mortality, death after specific number of CVD events (Death | CVD state-i.e. no difference in mortality after first, second or third CVD event was observed between groups) and progression of peripheral neuropathy

and Diamicron MR Controlled Evaluation) trial examining a combined approach of intensified blood pressure lowering and intensive glucose control [23] as well as from registry-based studies [2, 10]. In addition, analysis of the relation between number of risk factors simultaneously in control and cardiovascular outcomes in the BARI-2D (Bypass Angioplasty Revascularization Investigation 2 Diabetes) trial found a clear beneficial effect of having more risk factors in control [24], further supporting the concept of multifactorial treatment.

Long-term beneficial effects of glucose-lowering treatment in reducing microvascular complications have been demonstrated beyond the duration of a clinical trial in both type 1 and type 2 diabetes $[20,25,26]$. Lipid-lowering treatment has proven longterm beneficial effects with regards to CVD reduction [6]. However, the beneficial effects on cardiovascular outcomes of
Table 2 Distribution of specific cardiovascular event types by treatment allocation. Bottom row is the sum of the combined primary endpoint and other-cause mortality

\begin{tabular}{|c|c|c|c|c|c|c|}
\hline \multirow[t]{2}{*}{ Event type } & \multicolumn{3}{|c|}{ Intensive-therapy group } & \multicolumn{3}{|c|}{ Conventional-therapy group } \\
\hline & $\begin{array}{l}\text { No. of } \\
\text { patients }\end{array}$ & $\begin{array}{l}\text { No. of first } \\
\text { events }\end{array}$ & $\begin{array}{l}\text { No. of } \\
\text { events }\end{array}$ & $\begin{array}{l}\text { No. of } \\
\text { patients }\end{array}$ & $\begin{array}{l}\text { No. of first } \\
\text { events }\end{array}$ & $\begin{array}{l}\text { No. of } \\
\text { events }\end{array}$ \\
\hline Death from CVD & 12 & 4 & 12 & 26 & 4 & 26 \\
\hline Death from other cause & 26 & 13 & 26 & 29 & 11 & 29 \\
\hline Myocardial infarction & 9 & 7 & 11 & 23 & 12 & 40 \\
\hline Stroke & 10 & 4 & 11 & 25 & 19 & 41 \\
\hline Amputation & 13 & 9 & 26 & 18 & 6 & 39 \\
\hline Cardiac revascularisation & 10 & 8 & 17 & 20 & 9 & 29 \\
\hline Peripheral revascularisation & 7 & 6 & 13 & 11 & 5 & 20 \\
\hline All non-fatal first events & 35 & 34 & 78 & 52 & 52 & 169 \\
\hline Recurrent non-fatal events & 19 & & & 34 & & \\
\hline Combined primary endpoint & 39 & 38 & 90 & 56 & 56 & 195 \\
\hline Total number of events & & 51 & 116 & & 67 & 228 \\
\hline
\end{tabular}

Bottom row is the sum of the combined primary endpoint and other-cause mortality 


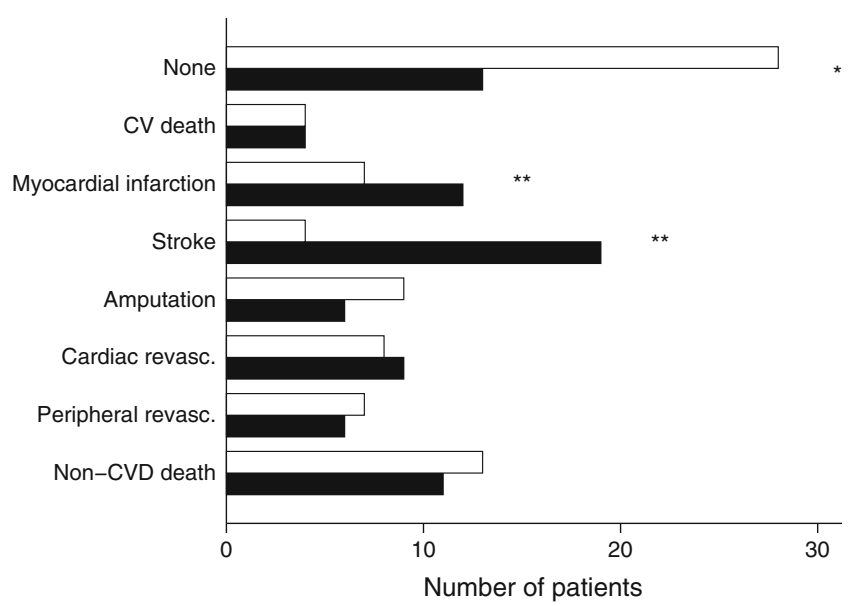

Fig. 4 Distribution of type of first event by treatment allocation. White bars, intensive-therapy patients; black bars, conventional-therapy patients. ${ }^{*} p<0.05$ and ${ }^{* *} p<0.01$ for difference between groups; revasc., revascularisation blood pressure- and glucose-lowering treatments seem to attenuate when treatment is relaxed post intervention [19, 21, 27]. Long-term beneficial effects in these trials have been termed 'metabolic memory' or 'legacy effect'. In these trials, intensified intervention according to protocol was stopped at the end of the trial and the subsequent risk factor control was relaxed or not reported. In contrast, patients in the Steno-2 study's intensivetherapy group continued treatment according to the original treatment goals while patients in the original conventional-therapy group started intensified treatment with identical targets during the follow-up period. Thus, from year 8 onwards all patients in both treatment arms in the Steno- 2 study received identical treatment with similar post-trial risk factor levels in the two groups. We interpret the continuing beneficial effects seen in the trial as a direct consequence of early intervention intensification in patients at lower absolute risk for late diabetic complications compared with a situation wherein increased vascular damage is
Fig. 5 Progression of microvascular complications. The black line is the smoothed survival estimate. Green areas under the curves depict the probability of being alive without (progression in) the specified microvascular complication and the different shades of orange represent progression to the specified progression state after the given follow-up duration. The lightly coloured areas above the black curve depict the fraction of patients who died after progression corresponding to the specified disease states Both the 'survival without progression' area (dark green) and the total 'survival' area (area under the black curve) are significantly larger in the intensive-therapy group for autonomic neuropathy, retinopathy and nephropathy (albuminuria) (i.e. the risk of disease progression is decreased for these outcomes in the intensive-therapy group). No significant difference in the progression of peripheral neuropathy between groups was observed. An example of interpretation: for autonomic neuropathy, it is shown that the fraction of patients that died with no progression is similar in the two groups (light green), but the fraction of patients who died after progression (light orange) was significantly larger in the conventional-therapy group

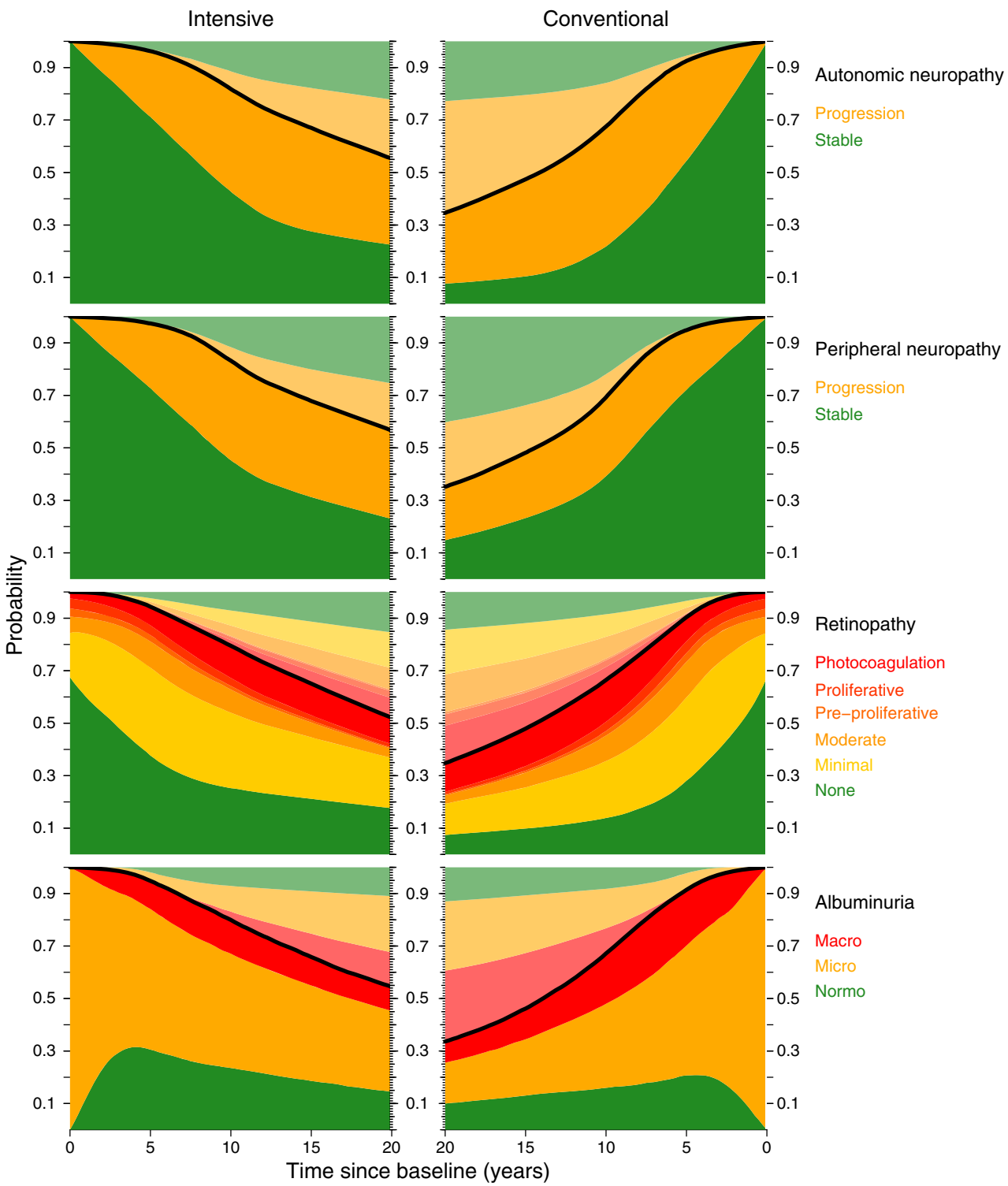


already present with intensification in later stages of the disease. This concept of early intervention in patients at lower risk has just proven beneficial for combined treatment of lipid and blood pressure lowering in patients at intermediate risk of, but without, CVD [28].

The Steno-2 study is robust in the sense that data on endpoints are considered complete due to the quality of relevant databases combined with low dropout rate and endpoint adjudication by an external expert committee blinded for treatment allocation. Additionally, the study design resembles a real-life situation, where researchers did not have direct influence on medicine compliance or lifestyle.

In conclusion, we found that intensified, multifactorial treatment of type 2 diabetes with microalbuminuria for 7.8 years compared with conventional treatment increases median life length by 7.9 years over 21.2 years of follow-up, and that these gained years were matched by years free from cardiovascular complications.

We must emphasise the significance of early, intensified risk factor control in patients with complicated type 2 diabetes. This approach is already broadly implemented according to clinical guidelines and the present findings should lead to even more focus on the potential preventive effects.

Acknowledgements We thank the patients who participated in the study and their families. I. Holstein, G. Lademann, B. B. Nielsen, M. Valerius and B. R. Jensen are thanked for their technical and managerial assistance (all Steno Diabetes Center, Gentofte, Denmark or Novo Nordisk Foundation Centre for Basic Metabolic Research, Copenhagen, Denmark). We thank J. Faber (Herlev Hospital, Denmark) and P. Hildebrandt (Frederiksberg Heart Clinic, Denmark) for their thorough examinations while serving on the endpoint committee and E. A. M. Gale (University of Bristol, UK) for critically reviewing the manuscript prior to submission.

Funding The study was funded by an unrestricted grant from Novo Nordisk A/S. Novo Nordisk A/S were not in any way involved in study design or in data collection, analysis or interpretation, but have had the opportunity to read and comment on the manuscript before submission.

Duality of interest $\mathrm{BC}$ is employed at Steno Diabetes Center, which is owned by Novo Nordisk A/S, and has equity share in Novo Nordisk A/S. $\mathrm{PR}$ has equity interest in Novo Nordisk A/S, research contracts with AbbVie, Novo Nordisk and Novartis and has received consulting honoraria from AstraZeneca, BMS, Boehringer Ingellheim, Eli Lilly, Novo Nordisk, Astellas and AbbVie) (all to institution). HLA is a consultant at Steno Diabetes Center. HHP has equity interest in Merck and has received consulting honoraria from $\mathrm{AbbVie}$ and Novartis. OP has equity interest in Novo Nordisk A/S. The Novo Nordisk Foundation Center for Basic Metabolic Research is an independent Research Center at the University of Copenhagen partially funded by an unrestricted donation from the Novo Nordisk Foundation (www.metabol.ku.dk). All other authors declare that there is no duality of interest associated with their contribution to this manuscript.

Contribution statement OP conceived and designed the original Steno- 2 study, devised and supervised the 21.2 years follow-up, acquired all funding throughout the study and provided key content to the manuscript. HHP contributed to the conception and supervision of the original Steno-2 study and provided key content to the manuscript. PG acquired all data up to the 13.3 years examination, handled patient care during the intervention period, planned the 21.2 years follow-up and supervised data acquisition and processing in the present follow-up. JO coordinated and performed the 21.2 years follow-up, acquired all data for the 21.2 years follow-up, supervised laboratory work and patient evaluations, processed data and performed statistical work. BC planned, conducted and supervised statistical work, provided additional analyses and figures, contributed to data interpretation and compiled the ESM. PR supervised and facilitated all work performed at Steno Diabetes Center (i.e. the patient assessments for the long-term follow-up), was responsible for quality assurance/quality control of the performed measures, provided key intellectual content to the discussion section and critically reviewed the manuscript. HLA provided and interpreted data regarding retinopathy, provided key intellectual content and critically reviewed the manuscript. The manuscript was drafted by PG and JO with contributions from all other authors. All authors gave final approval for the paper to be published. OP, HHP and PG are guarantors of this work.

Open Access This article is distributed under the terms of the Creative Commons Attribution 4.0 International License (http:// creativecommons.org/licenses/by/4.0/), which permits unrestricted use, distribution, and reproduction in any medium, provided you give appropriate credit to the original author(s) and the source, provide a link to the Creative Commons license, and indicate if changes were made.

\section{References}

1. Inzucchi SE, Bergenstal RM, Buse JB et al (2015) Management of hyperglycemia in type 2 diabetes, 2015: a patient-centered approach. Update to a position statement of the American Diabetes Association and the European Association for the Study of Diabetes. Diabetologia 58:429-442

2. Tancredi M, Rosengren A, Svensson AM et al (2015) Excess mortality among persons with type 2 diabetes. N Engl J Med 373:1720-1732

3. Cannon CP, Blazing MA, Giugliano RP et al (2015) Ezetimibe added to statin therapy after acute coronary syndromes. N Engl J Med 372:2387-2397

4. LaRosa JC, Deedwania PC, Shepherd J et al (2010) Comparison of 80 versus $10 \mathrm{mg}$ of atorvastatin on occurrence of cardiovascular events after the first event (from the Treating to New Targets [TNT] trial). Am J Cardiol 105:283-287

5. Murphy SA, Cannon CP, Wiviott SD, McCabe $\mathrm{CH}$, Braunwald E (2009) Reduction in recurrent cardiovascular events with intensive lipid-lowering statin therapy compared with moderate lipidlowering statin therapy after acute coronary syndromes from the PROVE IT-TIMI 22 (Pravastatin or Atorvastatin Evaluation and Infection Therapy-Thrombolysis In Myocardial Infarction 22) trial. J Am Coll Cardiol 54:2358-2362

6. Ford I, Murray H, Packard CJ et al (2007) Long-term follow-up of the West of Scotland coronary prevention study. N Engl J Med 357: $1477-1486$

7. Nissen SE (2009) Cardiovascular outcomes in randomized trials: should time to first event for "hard" end points remain the standard approach? J Am Coll Cardiol 54:2363-2365

8. Tikkanen MJ, Szarek M, Fayyad R et al (2009) Total cardiovascular disease burden: comparing intensive with moderate statin therapy insights from the IDEAL (incremental decrease in end points through aggressive lipid lowering) trial. J Am Coll Cardiol 54:2353-2357

9. Faerch K, Carstensen B, Almdal TP, Jorgensen ME (2014) Improved survival among patients with complicated type 2 diabetes in Denmark: a prospective study (2002-2010). J Clin Endocrinol Metab 99:E642-E646 
10. Gregg EW, Li Y, Wang J et al (2014) Changes in diabetes-related complications in the United States, 1990-2010. N Engl J Med 370: $1514-1523$

11. Gæde P, Vedel P, Parving HH, Pedersen O (1999) Intensified multifactorial intervention in patients with type 2 diabetes mellitus and microalbuminuria: the steno type 2 randomised study. Lancet 353: 617-622

12. Gæde P, Vedel P, Larsen N, Jensen GV, Parving HH, Pedersen O (2003) Multifactorial intervention and cardiovascular disease in patients with type 2 diabetes. N Engl J Med 348:383-393

13. Gæde P, Lund-Andersen H, Parving HH, Pedersen O (2008) Effect of a multifactorial intervention on mortality in type 2 diabetes. N Engl J Med 358:580-591

14. Ryden L, Grant PJ, Anker SD et al (2013) ESC guidelines on diabetes, pre-diabetes, and cardiovascular diseases developed in collaboration with the EASD: the Task Force on diabetes, pre-diabetes, and cardiovascular diseases of the European Society of Cardiology (ESC) and developed in collaboration with the European Association for the Study of Diabetes (EASD). Eur Heart J 34:3035-3087

15. American Diabetes A (2015) (8) Cardiovascular disease and risk management. Diabetes Care 38(Suppl):S49-57

16. Lynge E, Sandegaard JL, Rebolj M (2011) The Danish National Patient Register. Scand J Public Health 39:30-33

17. Pedersen CB (2011) The Danish Civil Registration System. Scand J Public Health 39:22-25

18. Gerstein HC, Miller ME, Genuth S et al (2011) Long-term effects of intensive glucose lowering on cardiovascular outcomes. N Engl J Med 364:818-828

19. Zoungas S, Chalmers J, Neal B et al (2014) Follow-up of bloodpressure lowering and glucose control in type 2 diabetes. $\mathrm{N}$ Engl $\mathrm{J}$ Med 371:1392-1406
20. Holman RR, Paul SK, Bethel MA, Matthews DR, Neil HA (2008) 10-year follow-up of intensive glucose control in type 2 diabetes. N Engl J Med 359:1577-1589

21. Holman RR, Paul SK, Bethel MA, Neil HA, Matthews DR (2008) Long-term follow-up after tight control of blood pressure in type 2 diabetes. N Engl J Med 359:1565-1576

22. Shi L, Ye X, Lu M et al (2013) Clinical and economic benefits associated with the achievement of both HbA1c and LDL cholesterol goals in veterans with type 2 diabetes. Diabetes Care 36:32973304

23. Zoungas S, de Galan BE, Ninomiya T et al (2009) Combined effects of routine blood pressure lowering and intensive glucose control on macrovascular and microvascular outcomes in patients with type 2 diabetes: new results from the ADVANCE trial. Diabetes Care 32: 2068-2074

24. Bittner V, Bertolet M, Barraza Felix R et al (2015) Comprehensive cardiovascular risk factor control improves survival: the BARI 2D trial. J Am Coll Cardiol 66:765-773

25. Lachin JM, Orchard TJ, Nathan DM, Group DER (2014) Update on cardiovascular outcomes at 30 years of the diabetes control and complications trial/epidemiology of diabetes interventions and complications study. Diabetes Care 37:39-43

26. Hayward RA, Reaven PD, Wiitala WL et al (2015) Follow-up of glycemic control and cardiovascular outcomes in type 2 diabetes. N Engl J Med 372:2197-2206

27. Advance Study Group (2016) Nine-year effects of 3.7 years of intensive glycemic control on cardiovascular outcomes. Diabetes Care 39:701-708

28. Yusuf S, Lonn E, Pais P et al (2016) Blood-pressure and cholesterol lowering in persons without cardiovascular disease. N Engl J Med 374:2032-2043 